Relations industrielles

Industrial Relations

\title{
P. Dionne et G. Ouellet, La communication interpersonnelle et organisationnelle: l'effet Palo-Alto
}

\section{Yvon Pépin}

Volume 46, numéro 3, 1991

URI : https://id.erudit.org/iderudit/050710ar

DOI : https://doi.org/10.7202/050710ar

Aller au sommaire du numéro

Éditeur(s)

Département des relations industrielles de l'Université Laval

ISSN

0034-379X (imprimé)

1703-8138 (numérique)

Découvrir la revue

Citer ce compte rendu

Pépin, Y. (1991). Compte rendu de [P. Dionne et G. Ouellet, La communication interpersonnelle et organisationnelle: l'effet Palo-Alto]. Relations industrielles / Industrial Relations, 46(3), 674-677. https://doi.org/10.7202/050710ar

Tous droits réservés (C Département des relations industrielles de l'Université Laval, 1991
Ce document est protégé par la loi sur le droit d'auteur. L'utilisation des services d'Érudit (y compris la reproduction) est assujettie à sa politique d'utilisation que vous pouvez consulter en ligne.

https://apropos.erudit.org/fr/usagers/politique-dutilisation/ 
«En fait, sur la base d'une dizaine d'études de postes, de différents niveaux et appartenant à différents services [lesquels?] des trois types d'institutions, nous pensons pouvoir conclure à l'existence de deux modèles. Le premier s'appliquerait aux personnels moins qualifiés des commissions scolaires où une certaine polyvalence serait recherchée de la part de ces personnels pour faire face à la complexification des emplois. Le second modèle serait celui retenu dans les cégeps, dans les universités où l'on tente de donner plus d'importance au groupe des techniciens et techniciennes, les croyant plus aptes à faire face aux nouvelles exigences des emplois.»

Une dizaine de postes étudiés qui relèvent de différents niveaux, de différents services et de trois types d'institutions, commissions scolaires, cégeps et universités. Voilà assurément un échantillon abondant!

Il faut cependant rendre justice à l'auteur ne serait-ce qu'en rapportant cette limite dont elle fait elle-même état, lorsqu'elle écrit à la page 142:

«Nous ne prétendons pas en arriver à des conclusions généralisables à l'ensemble du secteur tertiaire au Québec.»

Mais alors, pourquoi le sous-titre général de l'ouvrage et pourquoi les titres apportés à l'étude des trois (sous) facteurs considérés, des titres qui par ailleurs, sont eux-mêmes autant de conclusions?

Une dernière remarque pour terminer:

Le «tertiaire» de toute évidence ne saurait se limiter aux banques (et aux caisses populaires), aux services téléphoniques, au gaz, à l'électricité, au secteur de l'enseignement. Qu'en est-il du transport, des établissements commerciaux, des chaînes de distribution alimentaire, de l'hôtellerie, de la restauration? Des emplois «McDonald», des emplois, nous dit-on, précaires, déqualifiés, sous-payés, mais qui permettent de gonfler des statistiques de l'emploi, si chères à nos politiciens?

Bernard SOLASSE

Université Laval

La communication interpersonnelle et organisationnelle: l'effet Palo-Alto, par P. Dionne et G. Ouellet, Boucherville (Québec), Gaëtan Morin éditeur, 1990, 144 p.,

ISBN 2-89105-334-6

Neuf ans après La gestion des équipes de travail publié chez Gaëtan Morin, en passant par la soutenance de leurs thèses de doctorat et de quelques articles portant sur la négociation collective, Dionne et Ouellet présentent, cette fois en co-édition franco-québécoise (Gaëtan Morin et Éditions d'organisation), un ouvrage plus général où ils précisent et développent le cadre théorique qui sert d'assise à leur pratique professionnele en consultation organisationnelle.

Ceux et celles qui ont lu le premier ouvrage constateront que les auteurs, même s'ils se situent toujours dans la perspective de la «pragmatique de la communication», l'exploitent avec beaucoup plus d'envergure, de précision, et de maîtrise. Il y a aussi progression dans un style d'écriture original qui déjà leur était caractéristique: simple et léger mais aussi sérieux et réfléchi, insolent et quelquefois mordant mais aussi profondément sensible aux vicissitudes de la condition humaine, avec, en filigrane, un humour qui ne se dément qu'en de rares occasions. 
Bref, ils ont amélioré non seulement la qualité du discours mais aussi celle du style et du «ton» pour le véhiculer. Et dans le cas de cet ouvrage, l'un ne va pas sans l'autre: l'expression de l'idéologie palo-altienne s'accommoderait mal du langage académique neutre et aseptisé habituel. Autre avantage: la lecture est d'autant plus agréable!

Tout en ayant une portée théorique importante et incontestable, l'ouvrage prend comme cible le caractère pratique et concret des problèmes de communication, mais en tant qu'ils sont générés par nos croyances spontanées en matière de communication. L'objectif, quoiqu'audacieux, est relativement simple: dans un premier temps, disqualifier systématiquement un certain nombre de ces croyances «spontanées» qui, selon les auteurs, nous réduiraient à l'impuissance et à la frustration; dans un deuxième temps, leur substituer un autre ensemble de croyances qui permettraient une marge de manoeuvre plus importante, un usage plus «émancipé» des processus communicationnels. Chemin faisant, les auteurs "contraignent» progressivement (et implicitement) le lecteur à se construire une image de la communication et fournissent ponctuellement (quelquefois à petites doses, quelquefois à dose massive) les concepts nécessaires pour transformer cette image en théorie articulée, palo-altienne bien entendu! Sur le plan strictement rhétorique et pédagogique, le procédé est très habile... et très paloaltien! Étant donné les dimensions à la fois explicite et implicite de l'objectif, l'ordre des chapitres est très adéquat.

Le premier, titré «Mise en contexte de la communication humaine: mythologie d'aujourd'hui et d'hier», entreprend, d'une façon plutôt générale, le sabotage d'une certaine vision traditionnelle de la communication, surtout dans ses avatars scientifiques. En soulignant les impasses générales auxquelles nous conduit cette perspective, on plante le décor pour les critiques plus précises à venir. Le ton est quelquefois désagréablement alarmiste (cette vision nous "condamnerait à la solitude éternelle", elle rendrait l'humain «incompréhensible à ses propres yeux») et caricatural (la distinction émetteur/récepteur «donne l'impression qu'on devient sourd lorsqu'on émet et muet quand on reçoit»), au point où on a quelquefois le réflexe de se porter au secours de cette bonne vieille maison familière traitée avec si peu de respect. Malgré ces protestations, le travail de sape fait lentement son effet et, à la fin du chapitre, on est prêt à en entendre plus sur cette drôle de façon d'envisager la communication.

Cependant, on devra attendre un peu plus tard. Le deuxième chapitre continue la critique en s'attaquant cette fois à 19 cibles stratégiques: des croyances spécifiques qui s'articulent pour former la vision globale attaquée en première partie. Ces «mythes» sont des «allants de soi» sur lesquels reposent nos comportements habituels et spontanés en communication: «il faut se parler pour se comprendre», "pour être compris, un message doit être formulé clairement», «une attitude objective est une panacée universelle aux divergences d'opinion», «la communication porte sur des faits communément perçus ou perceptibles», "on est toujours convaincu par les meilleurs arguments», «on peut mettre fin à une relation en refusant de communiquer», etc. En fait, je crois que ces 19 mythes-cibles n'en recouvrent que 3 ou 4 qui seraient attaqués à plusieurs reprises sous des angles différents, histoire de s'assurer des dommages. L'énumération est agaçante (une lecture plus classique pourrait légitimement considérer ceci comme une faiblesse théorique: un regroupement théorique possible n'a pas été fait, aux dépens de l'économie conceptuelle), mais somme toute l'effet d'ensemble du chapitre parle de lui-même: dommage il y a! Quand la cible n'est pas atteinte sous un angle, elle l'est sous l'autre. Et à chaque fois, on justifie le bien-fondé de l'attaque et on propose des croyances de rechange qui, promotion oblige, seraient moins mensongères, moins erronées, moins mythiques, plus adéquates comme représentations de la réalité de la communication. En fait la critique permet aux auteurs de formuler implicitement une «image corrigée» de la réalité communicationnelle susceptible de mettre en valeur l'utilité du cadre conceptuel présenté dans le chapitre suivant. 
Le chapitre 3, qualifié de "parenthèse théorique», présente en effet une charpente conceptuelle qui rend cohérentes à la fois la critique qui précède et les implications pratiques qui sui, vent. Ces propositions théoriques (deux axiomes et six corollaires) sont minimales, en ce sens qu'elles s'en tiennent à l'essentiel. Les lecteurs qui ne connaissaient pas l'approche de PaloAlto seront tout juste renseignés (seulement deux axiomes originaires sont employés, bien cadrés cependant par une présentation "magistrale» de la notion cruciale d'«accord pragmatique»). Ceux qui connaissent l'approche seront tentés de crier à la réduction et à la simplification, mais seront probablement intéressés par la présence d'altérations/ajouts à la formulation originale (les 6 corollaires). Dionne et Ouellet campent carrément la communication comme une négociation perpétuelle non seulement de l'identité des participants mais aussi de la construction de la réalité à laquelle ils doivent s'adapter. L'utilité des axiomes et corrélaires est illustrée par l'analyse d'une anecdote où un enfant «triomphe» d'un adulte (le pouvoir des opprimés!), bel exemple du pouvoir émancipateur de leur version de la communication. Tout est maintenant en place pour formuler en détail ces possibilités d'émancipation.

C'est l'entreprise du chapitre 4: pour chaque mythe dénoncé, une avenue stratégique émancipatrice est proposée qui consiste à «s'adapter aux effets de la communication plutôt que d'essayer de la contrôler» (selon la version des auteurs) ou «à se comporter systématiquement de façon contraire à ce que dicte le «bon sens» ou le «sens commun» (selon la première impression du lecteur néophyte). On se fait prescrire comme parfaitement adéquats des comportements de communication pour le moins étranges tels que: «jouer sur l'implicite (et ne surtout pas l'expliciter)», «interpréter le non-dit (peu importe la justesse de cette interprétation)», «entretenir le problème et le conflit (plutôt que tenter de les résoudre)", "semer la confusion et le doute (plutôt que tenter de clarifier la situation)», «se comporter selon une vision inédite de la réalité (plutôt que conformément à une vision supposée commune)", "s'abstenir d'intervenir dans des moments cruciaux», etc. Ces comportements ne sont toutefois étranges que sous le regard de l'ancienne mythologie, et leur utilité devient transparente pour le lecteur qui adopte la «mythologie stratégique» des auteurs et qui considère avec eux que l'enjeu de la communication est non l'harmonie et le bien-être collectif, mais la (dure) négociation de la réalité et de ses implications pour les participants. Les anecdotes qui illustrent ponctuellement cette viabilité sont d'ailleurs souvent savoureuses, bien qu'elles soient quelquefois discutables, d'un goût douteux, et qu'elles souffrent presque systématiquement de «triomphalisme aigü» (promotion oblige, la nouvelle grille doit expliquer même les échecs mais les auteurs préfèrent rapporter leurs exploits). En recadrant la situation, la «victime» initiale ou probable de la communication tire son épingle du jeu et la «nouvelle victime» ne souffre même pas de son incapacité à utiliser l'information stratégique disponible. Elle est dupe de son propre bonheur!

La deuxième partie de ce chapitre utilise le même procédé, mais de façon "positive»: il s'agit d'exploiter non plus la foi des autres dans les anciens mythes, mais bien sa propre foi dans les «méta-énoncés stratégiques» (corollaires) énoncés en chapitre 3. Cette partie, titrée «La puissance des nouveaux mythes», illustre, d'une façon quelquefois tout aussi agaçante, la valeur stratégique d'une manipulation consciente de la communication à partir des nouvelles croyances proposées dans l'ouvrage.

La conclusion est particulièrement intéressante, en ce sens qu'elle met en évidence le talon d'Achille des auteurs. Il s'agit ni plus ni moins d'un "méta-commentaire» où ils tentent d'inclure leur propos dans leur propre théorie: ce volume est lui aussi une communication et tout ce qui y a été dit à propos de la communication s'applique au message qui y est véhiculé. Tout ce qui précède constitue donc un exercice de promotion d'une version de la réalité de la communication, celle des auteurs. La nouvelle mythologie n'est donc pas plus vraie, plus adéquate, plus juste (comme on le laissait supposer plus haut à des fins rhétoriques), que l'ancienne. Elle pourrait tout au plus s'avérer plus viable selon le but visé. Mais là encore, seul le lecteur peut en décider (et assumer les implications de sa décision). 
Dans ce contexte, $\mathrm{j}$ 'ai de la difficulté à prendre au sérieux «l'aveu», en fin de parcours (histoire d'être honnête) de 24 propositions sur la réalité de la communication qui, semble-t-il, auraient guidé toute l'entreprise. J'aurais préféré que la disqualification des anciens mythes tout au long du parcours se fasse méthodiquement en termes de leur viabilité et non en termes d'erreur, de fausseté, d'arbitraire, d'étroitesse de vue, de manque de perspective. En plus de rendre le message ambigü, cette rhétorique de disqualification n'a rien d'original, et peut-être rien de palo-altien!

Par contre je partage, dans une perspective pragmatique, un point de vue mis en évidence en conclusion: la définition de la réalité en général et de la réalité de la communication en particulier est une question de foi, de choix, et de but sur le plan personnel, et d'effet sur le plan interpersonnel. Le problème consiste à assumer les effets de ses choix, ce qui n'est pas toujours évident! Si votre but en étudiant la communication est de soumettre les autres (et de vous soumettre vous-même) à une version univoque de la réalité, oubliez l'approche de Palo-Alto; si par contre vous recherchez des stratégies pour vous soustraire au réel des autres, vous pouvez y trouver des avenues intéressantes, quoique limitées. Il faudra cependant payer le "fun" associé à la vision ludique de l'interaction sociale présentée par Dionne et Ouellet en renonçant à des certitudes et à une certaine conception du succès en communication.

De plus, la pratique de cette façon d' «être en communication» est loin d'être facile. La pragmatique de la communication n'est pas un ensemble de recettes-miracles. Et celà les auteurs, préoccupés de la promotion de leur point de vue, ne le soulignent peut-être pas assez. Par exemple, l'intérêt des «avenues stratégiques» du chapitre 4 est selon moi beaucoup plus d'ordre théorique (comme éléments exemplaires d'une façon alternative de concevoir et de vivre la communication quotidienne) que d'ordre pratique ou technique (comme façons ponctuelles de "résoudre" des problèmes singuliers de communication). En ce sens, je crois que l'ouvrage vaut plus par l'impression d'ensemble qu'il laisse après la lecture que par les analyses détaillées de différents aspects de la communication. On aura intérêt à voir ces dernières comme les éléments d'un message global.

En conclusion, je crois que cet ouvrage saura susciter l'intérêt de toute personne qui s'interroge sur les difficultés de la communication quotidienne, qu'elle soit initiée ou non à l'approche de Palo-Alto. Son style (et son propos) provocateur pourra séduire les uns et exaspérer les autres, mais je serais surpris que ce livre laisse la lectrice ou le lecteur indifférent. Le titre, cependant, peut induire en erreur: celui ou celle qui y cherche une théorie de la communication organisationnelle comme telle peut être déçu/e. Il s'agit avant tout d'une approche à la communication quotidienne telle qu'elle est vécue à différents niveaux d'interaction, dont le niveau organisationnel. On retrouve, bien sûr, beaucoup d'exemples de difficultés de communication dans l'organisation, mais peu ou pas de propos théoriques sur la communication organisationnelle comme telle. Ceci s'explique en partie par le choix épistémologique des auteurs qui leur interdit de se prononcer sur des systèmes autrement que comme organisations perceptuelles d'individus. L'organisation «réelle» existe peut-être, mais elle n'a d'effet qu'en tant qu'elle est perçue par quelqu'un. Encore là, certains/es protesteront!

Yvon PÉPIN

Université Laval 